\title{
Dynamic Modeling and Analysis of Demagnetizing Rotor of Permanent Magnet Synchronous Motor
}

\author{
Bifeng Zhou $\mathbb{D}^{1,2}$ Guoning Tang, ${ }^{1}$ and Yiping $\mathrm{Luo}^{3}$ \\ ${ }^{1}$ School of Mechanical Engineering, Hunan University of Science and Technology, Xiangtan 411201, China \\ ${ }^{2}$ Hunan Electrical College of Technology, Xiangtan 411101, China \\ ${ }^{3}$ College of Electrical Information, Hunan Institute of Engineering, Xiangtan 411101, China \\ Correspondence should be addressed to Bifeng Zhou; zhoubifeng99@163.com
}

Received 15 December 2020; Revised 29 March 2021; Accepted 30 April 2021; Published 13 May 2021

Academic Editor: Dario Richiedei

Copyright ( $\odot 2021$ Bifeng Zhou et al. This is an open access article distributed under the Creative Commons Attribution License, which permits unrestricted use, distribution, and reproduction in any medium, provided the original work is properly cited.

\begin{abstract}
This study performs a dynamic modeling and analysis of the axial uniform demagnetization rotor of a motor to investigate the demagnetization peculiar for permanent magnet synchronous motor (PMSM). First, the air-gap change in the motor is analyzed by constructing a dual coordinate system of stator and rotor, and the unbalanced magnetic pull (UMP) model under uniform demagnetization and eccentricity is constructed. Second, combined with the UMP and the self-gravity, a dynamic model of the rotor under a uniform demagnetization and eccentricity is established. Lastly, the accuracy of the mathematical model of UMP under uniform demagnetization and eccentricity is confirmed by the mutual corroboration of Maxwell simulation and MATLAB calculation; and based on the dynamics model, the dynamic characteristics of the rotor system under different degrees of uniform demagnetization are studied. This study provides a theoretical basis for the accurate demagnetization fault diagnosis and vibration control of PMSMs in the future.
\end{abstract}

\section{Introduction}

The stability of permanent magnet materials for permanent magnet synchronous motors (PMSMs) is affected by temperature, magnetic field, chemical factors, and time. Considering the actual working condition environment of PMSMs and according to research on the demagnetization mechanism of PMSMs [1-3], the demagnetization of PMSMs is inevitable. Substantial research on the demagnetization of PMSMs has focused on the control of the motor and the performance of the PMSM after demagnetization. Such research has covered the permanent magnet flux linkage of PMSMs [4-7], the fault-tolerant control of PMSM demagnetization [8-11], and the finite element modeling of PMSM demagnetization [12-14].

However, PMSMs often exist as a key component in practical industrial systems, such as permanent magnet synchronous wind generators. Not only does the demagnetization of PMSMs affect the performance of the motor itself, but the vibration of the motor due to demagnetization also considerably affects the system where the motor is located. Thus, studying the demagnetization vibration of PMSMs is crucial.

Existing studies on the demagnetization vibration of PMSMs are relatively rare. Xiang [15] analyzed the stiffness characteristics of the rotor system of the PMSM and investigated the nonlinear dynamic behavior influenced by the unbalanced magnetic pull (UMP) based on the Jeffcott rotor model. Liu [16] investigated the nonlinear oscillations of a PMSM based on a Jeffcott rotor-bearing system and considered the effects of the UMP, the nonlinear restoration forces due to the Hertz contact force and bearing clearance, the rotor weight, and the rotor mass eccentricity.

Some scholars have studied the demagnetization vibration of PMSMs and achieved promising results. However, the existing research results only consider the UMP generated by PMSM demagnetization and do not consider the specific demagnetization form of the permanent magnet of the PMSM. That is, only the demagnetization phenomenon is considered, and the demagnetization state is not quantitatively described. Therefore, the resulting dynamic model does not consider the demagnetization quantitatively. 
Motivated by the above analysis, this study focuses on the dynamic modeling and analysis of the demagnetizing rotor of PMSMs. First, the uniform demagnetization state of permanent magnets is quantitatively described by the demagnetization angle and the decrease of air-gap magnet motive force (MMF) at the demagnetization angle (air-gap MMF drop percentage); on the basis of this description, the air-gap change in the motor is analyzed by constructing a dual coordinate system of stator and rotor, and the unbalanced magnetic pull (UMP) model under uniform demagnetization and eccentricity is constructed. Second, combined with the UMP and the self-gravity, a dynamic model of the rotor under a uniform demagnetization and eccentricity is established. Lastly, the accuracy of the mathematical model of UMP under uniform demagnetization and eccentricity is confirmed by the mutual corroboration of Maxwell simulation and MATLAB calculation, and based on the dynamics model, the dynamic characteristics of the rotor system under different degrees of uniform demagnetization are studied. This study provides a theoretical basis for the accurate demagnetization fault diagnosis and vibration control of PMSMs in the future.

\section{Nonlinear Dynamic Model of a Rotor Under Demagnetization}

2.1. Dynamical Model of a Rotor System. The demagnetization of the permanent magnet in the rotor of the permanent magnet synchronous motor (PMSM) results in an uneven air-gap flux density in the motor, resulting in unbalanced magnetic pull (UMP) acting on the rotor, which makes the rotor eccentricity, and the eccentricity of the rotor further changes the air-gap flux density of the motor, thereby affecting the dynamic characteristics of rotor operation.

Figure 1 shows a schematic of rotor eccentricity under demagnetization. $O$ and $O^{\prime}$ are the geometric centers of the stator and rotor, respectively; $\varepsilon(t)$ is the eccentricity between the geometric center of the rotor and the stator; $R$ is the radius of the rotor; $\delta_{0}$ is the average of air-gap length; and $\delta(\psi)$ is the length of the air-gap corresponding to angle $\psi$ in the rotating coordinate system $x^{\prime} O^{\prime} y^{\prime}$.

To establish the rotor dynamic model of a PMSM under demagnetization, this study assumes the following:

(1) All damping is considered as linear viscous damping in the calculation process, and disregard the effects of nonlinearity; the concentrated mass method is adopted without considering the axial motion of each component [17].

(2) The case where the rotor's permanent magnet is axially uniformly demagnetized is considered. The combined amount of changes in the corresponding air-gap magnet motive force (MMF) caused by demagnetization in any axially uniform state of the permanent magnet can be described by the following two parameters: demagnetization angle and the decrease of air-gap MMF at the demagnetization angle (air-gap MMF drop percentage).

On the basis of the above assumptions, the dynamic model of the magnetic field-rotor coupling system can be obtained.

$$
\left\{m \ddot{x}(t)+c_{x x} \dot{x}(t)+k_{x x} x(t)=F_{M x}(t), m \ddot{y}(t)+c_{y y} \dot{y}(t)+k_{y y} y(t)=F_{M y}(t)-m g,\right.
$$

where $x, y, \dot{x}, \dot{y}, \ddot{x}, \ddot{y}$ are the displacement, velocity, and acceleration of the rotor in the $x$ and $y$ axes, respectively; $m$ is the structural quality of the rotor; $c_{x x}, c_{y y}$ are the operational damping in the $x$ and $y$ directions; $k_{x x}, k_{y y}$ are the stiffness of the rotor in the positive directions of the $x$ and $y$ axes; and $F_{M x}(t)$ and $F_{M y}(t)$ are the UMP in the $x$ and $y$ directions, respectively.

2.2. Modeling of Demagnetization. This section analyzes $F_{M x}(t)$ and $F_{M y}(t)$ mentioned in the previous section.

2.2.1. Air-Gap Flux Density. The demagnetization of permanent magnet in the rotor of permanent magnet synchronous motor (PMSM) results in unbalanced magnetic pull (UMP), which makes the rotor eccentric. Figure 2 shows a cross section diagram of rotor demagnetization. With the center $O^{\prime}$ of the rotor taken as the origin, the rotor rectangular coordinate system $x^{\prime} O^{\prime} y^{\prime}$ is established. In such system, the $x^{\prime}$-axis direction is opposite to the center line direction of the demagnetization angle, and the coordinate system rotates with the rotor. With the center of the stator $O$ taken as the origin, the stator stationary coordinate system is constructed with the initial direction of the $x^{\prime}$ and $y^{\prime}$ coordinate axes in the coordinate system $x^{\prime} O^{\prime} y^{\prime}$ of the rotor. Thus, the initial stator and rotor coordinate systems are parallel.

The displacement variation of eccentricity between the stator and rotor of a PMSM is shown in Figure 2. $\beta$ is half of demagnetization angle, $\psi$ is the space angle associated with the $x^{\prime}$ axis in the rotating coordinate system $x^{\prime} O^{\prime} y^{\prime}, \delta_{0}$ is the average air-gap length of the PMSM, and $\varepsilon(t)$ is the eccentricity scale of the rotor, and $\theta(t)$ is the angle between the connecting line of the stator and rotor center $O, O^{\prime}$ and the $x$ axis. When the rotor runs, it satisfies

$$
\theta(t)=\omega t+\theta_{0}
$$

where $\theta_{0}$ is the angle between the resultant force of the rotor and the $x^{\prime}$ axis in the rotating coordinate system $x^{\prime} O^{\prime} y^{\prime}$ and $\omega$ is the rotational angular velocity of the rotor.

$\xi(t)=\left(\varepsilon(t) / \delta_{0}\right)$ is set as the eccentricity ratio of the rotor. In the rotating coordinate system $x^{\prime} O^{\prime} y^{\prime}$, the rotor eccentricity caused by the demagnetization of the permanent magnet is considered, and the air-gap distribution $\delta(\psi, t)$ can be approximately expressed as follows [18]: 


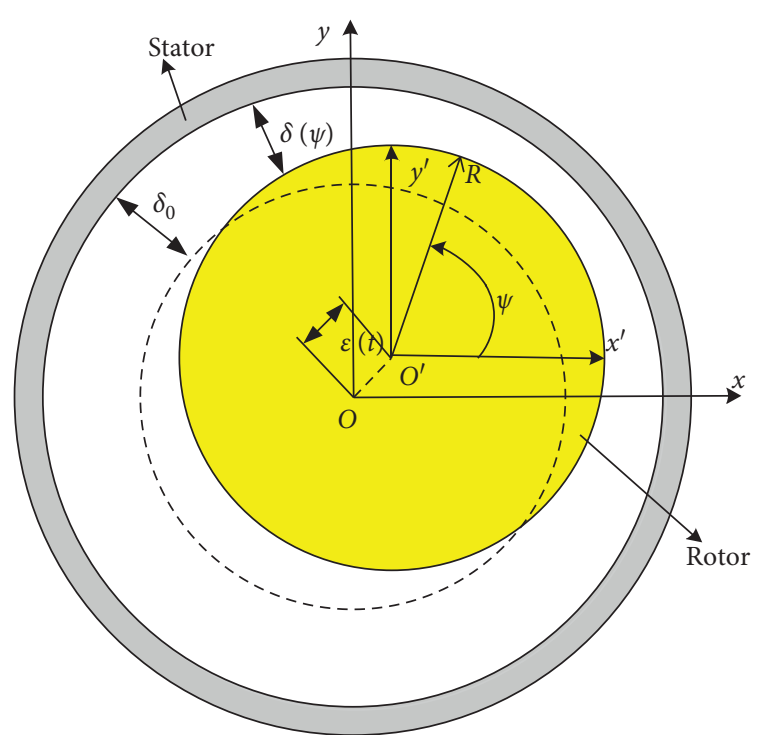

FIgURE 1: The schematic diagram of rotor eccentricity under demagnetization.

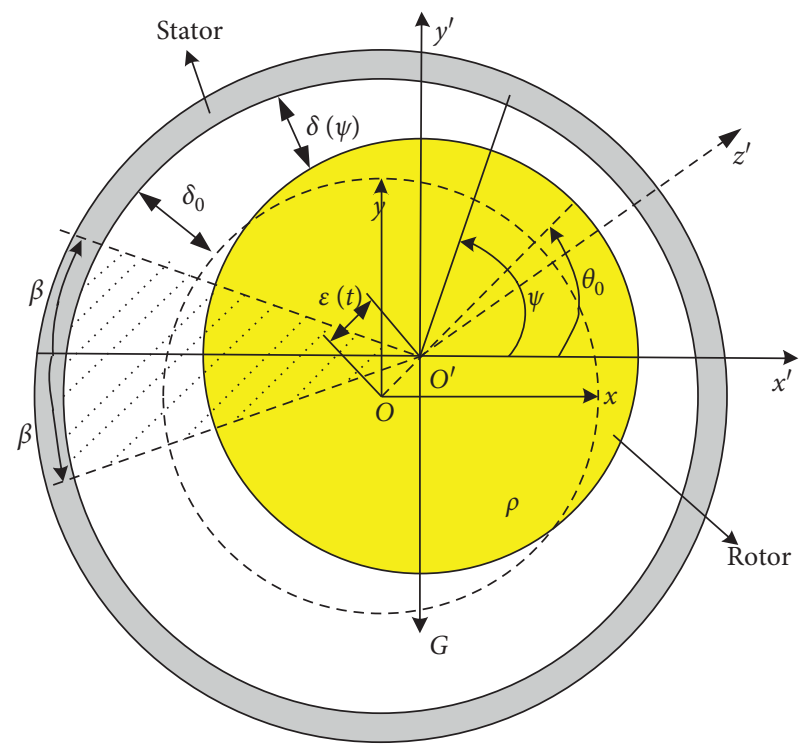

Figure 2: The cross-section diagram of rotor demagnetization.

$$
\begin{aligned}
\delta(\psi, t) & \approx \delta_{0}-\varepsilon(t) \cos \left(\psi-\theta_{0}\right) \\
& =\delta_{0}\left[1-\xi(t) \cos \left(\psi-\theta_{0}\right)\right] \\
& =\delta_{0}-x^{\prime}(t) \cos (\psi)-y^{\prime}(t) \sin (\psi),
\end{aligned}
$$

where $x^{\prime}(t)=\varepsilon(t) \cos \left(\theta_{0}\right)$ and $y^{\prime}(t)=\varepsilon(t) \sin \left(\theta_{0}\right)$ are the projection displacements of the eccentric vector in the $x^{\prime} O^{\prime} y^{\prime}$ coordinate system. The permeability of the air gap is as follows:

$$
\begin{aligned}
\Lambda(t) & =\frac{\mu_{0}}{\delta(\psi, t)} \approx \frac{\mu_{0}}{\delta_{0}}\left[1+\xi(t) \cos \left(\psi-\theta_{0}\right)\right] \\
& =\frac{\mu_{0}}{\delta_{0}^{2}}\left[\delta_{0}+x^{\prime}(t) \cos (\psi)+y^{\prime}(t) \sin (\psi)\right],
\end{aligned}
$$

where $\mu_{0}$ is the permeability of vacuum.
Considering the demagnetization of the permanent magnets of a PMSM, the fundamental wave of the air-gap MMF established by the current in the torque winding and the permanent magnet field in the rotor of the PMSM is as follows [18]:

$$
F(\psi, t)=F_{\delta} \cos (w t-p \psi-u)
$$

where $p$ is the number of pole pairs of the torque winding, $F_{\delta}$ is the amplitude of the fundamental wave of the air-gap MMF, $u$ is the spatial initial phase angle of the fundamental wave of the air-gap MMF, and $w$ is the angular frequency of the winding current. The rotation angular velocity $\omega$ of the rotor and the angular frequency $w$ of the winding current satisfy $\omega=(60 * 2 * \pi * w / p)$.

In the rotating coordinate system $x^{\prime} O^{\prime} y^{\prime}$ and in consideration of the axial direction uniform demagnetization of the rotor of a PMSM, the change of the amplitude of the airgap MMF caused by the demagnetization of the permanent magnet is equivalently mapped to demagnetization angle $\beta$ on both sides of the coordinate $-x^{\prime}$ axis, and the decrease of air-gap MMF drops to $(1-\zeta) * \widehat{F}$ (at the demagnetization angle). At this time, the amplitude of the fundamental wave of the air-gap MMF $F_{\delta}$ is

$$
F_{\delta}= \begin{cases}\widehat{F}, & \psi \in(-\pi+\beta, \pi-\beta), \\ (1-\zeta) \widehat{F}, & \psi \in(-\pi,-\pi+\beta) \cup(\pi-\beta, \pi),\end{cases}
$$

where $\beta \in(0, \pi)$ and $\zeta \leqslant 1$ is the decrease of air-gap MMF at the demagnetization angle (air-gap MMF drop percentage).

In the rotating coordinate system $x^{\prime} O^{\prime} y^{\prime}$, in consideration of the local demagnetization of the permanent magnet of a PMSM, the air-gap flux density in the motor is as follows:

$$
\begin{aligned}
B(\psi, t)= & \Lambda(\psi, t) F(\psi, t) \\
= & \frac{\mu_{0}}{\delta_{0}^{2}} F_{\delta}\left[\delta_{0}+x^{\prime}(t) \cos (\psi)+y^{\prime}(t) \sin (\psi)\right] \\
& \cos (w t-p \psi-u) .
\end{aligned}
$$

Assuming that the permanent magnet is in normal condition and the air gap is uniform, the amplitude of flux density of air-gap for pair pole $p$ is [18]

$$
\begin{aligned}
\widehat{B} & =\frac{\mu_{0}}{\delta_{0}} \widehat{F} \\
& =\frac{\mu_{0}}{\delta_{0}}\left(\frac{3}{2} \frac{4}{\pi} \frac{N I}{2 p}\right),
\end{aligned}
$$

where $N$ is the number of turns of each phase of the torque winding and $I$ is the amplitude of the excitation current of the torque winding.

2.2.2. Analysis of UMP. The magnetic tension formed on the boundary of a magnetic material with varying permeability (such as air and core) in the magnetic circuit of a PMSM is called Maxwell force, also known as detent force; the 
direction of the force is perpendicular to the boundary surface of the magnetic material.

When analyzing the magnetic field of the air gap of a PMSM, we assume that the magnetic density of the air gap is symmetrically distributed. At this time, the resultant force of the Maxwell force is zero. However, considering the local demagnetization of the permanent magnet in PMSM, the magnetic density distribution in the air gap of the motor is not uniform. Thus, the resultant Maxwell force is not zero.

The rotor of a PMSM is subjected to the Maxwell force of the nonzero resultant force; as such, the rotor produces eccentricity, and the magnetic density in the air gap of the PMSM changes, further altering the resultant Maxwell force. The larger the eccentricity of the rotor, the greater the Maxwell force.

In accordance with the Maxwell tensor method, the magnetic density in the air gap of a PMSM is set as $B$, and the Maxwell force acting on the $\mathrm{d} S$ area of the rotor surface is as follows:

$$
\mathrm{d} F_{M}=\frac{B^{2} \mathrm{~d} S}{2 \mu_{0}} .
$$

Then the component of Maxwell force along the $x, y$ direction is as follows:

$$
\begin{aligned}
\mathrm{d} F_{M x}(\psi) & =\frac{B^{2}(\psi, t) l_{0} r \mathrm{~d} \psi}{2 \mu_{0}} \cos (\psi), \\
\mathrm{d} F_{M y}(\psi) & =\frac{B^{2}(\psi, t) l_{0} r \mathrm{~d} \psi}{2 \mu_{0}} \sin (\psi),
\end{aligned}
$$

where $B(\psi, t)$ is the distribution of magnetic density in the air gap; $l_{0}$ is the effective core length of the rotor; $r$ is the external diameter of the rotor; and $\psi$ is the space angle.

By substituting equation (7) into equations (10) and (11), the integral operation of equations (10) and (11) is completed, and the Maxwell force in the $x^{\prime}, y^{\prime}$ direction can be obtained under the rotor eccentricity caused by the local demagnetization of the permanent magnet of the PMSM:

$$
\begin{aligned}
& F_{M x^{\prime}}(t)=\int_{0}^{2 \pi} \eta \kappa \frac{l_{0} r}{2 \mu_{0}}\left(\frac{\mu_{0}}{\sigma_{0}^{2}} F_{\sigma}\right)^{2}\left[\sigma_{0}+\gamma\left(x^{\prime}(t) \cos (\psi)+y^{\prime}(t) \sin (\psi)\right)\right]^{2} \cos ^{2}(w t-p \psi-u) \cos \psi \mathrm{d} \psi, \\
& F_{M y^{\prime}}(t)=\int_{0}^{2 \pi} \eta \kappa \frac{l_{0} r}{2 \mu_{0}}\left(\frac{\mu_{0}}{\sigma_{0}^{2}} F_{\sigma}\right)^{2}\left[\sigma_{0}+\gamma\left(x^{\prime}(t) \cos (\psi)+y^{\prime}(t) \sin (\psi)\right)\right]^{2} \cos ^{2}(w t-p \psi-u) \sin \psi \mathrm{d} \psi,
\end{aligned}
$$

where $F_{M x^{\prime}}(t)$ and $F_{M y^{\prime}}(t)$ are UMPs in the direction of the $x^{\prime}$ and $y^{\prime}$ coordinate axes in the rotating coordinate system $x^{\prime} O^{\prime} y^{\prime}$. Initially, the angle between the coordinate axis of the rotating coordinate system $x^{\prime} O^{\prime} y^{\prime}$ and that of stationary coordinate system $x \mathrm{O} y$ is zero. During the operation of the rotating coordinate system $x^{\prime} O^{\prime} y^{\prime}$, the angle between the two coordinate systems is related to the rotational speed of the rotor, satisfying $x=x^{\prime} \cos (\omega t), y=x^{\prime} \sin (\omega t)$, $y=y^{\prime} \cos (\omega t)$, and $x=-y^{\prime} \sin (\omega t)$. Under stationary coordinate system $x O y$, the UMP is as follows:

$$
\begin{aligned}
& F_{M x}=F_{M x^{\prime}}(t) \cos (\omega t)-F_{M y^{\prime}}(t) \sin (\omega t), \\
& F_{M y}=F_{M x^{\prime}}(t) \sin (\omega t)+F_{M y^{\prime}}(t) \cos (\omega t) .
\end{aligned}
$$

Note 1. Considering the actual operation of the motor and air-gap flux density loss and other factors, set three parameters of $\eta, \kappa, \gamma$ in the UMP model (12), (13). For different types of PMSM, the three parameters $\eta, \kappa, \gamma$ are different. Among them, in the UMP model (12), (13), $\eta$ is the utilization coefficient of air-gap flux density; $\kappa$ is the influence coefficient of demagnetization on air-gap flux density of motor, when the PMSM is normal, $\kappa=1$. The $\gamma$ coefficient is related to the change of air-gap flux density caused by rotor eccentricity.
Note 2. Equations (12) and (13) are integral formulas, which shows variable $F_{\delta} . F_{\delta}$ is a piecewise function in equation (6) which is related to $\psi$, and so equations (12) and (13) are also an integral function related to $\psi$. In the process of integrating equations (12) and (13), the piecewise change of the demagnetization function $F_{\delta}$ should be considered.

Note 3. Based on equations (12) and (13), consider the rotor speed, and perform coordinate transformation on $F_{M x^{\prime}}(t)$ and $F_{M y^{\prime}}(t)$. The equations (14) and (15) are the UMP of the rotor under the directions $O x$ and $O y$ in the rectangular coordinate system $x \mathrm{O} y$ of the stator. Therefore, in equation $(1), F_{M x}(t)$ and $F_{M y}(t)$ are functions related to $x(t), y(t)$ and $F_{\delta}$. In our research, the demagnetization of the permanent magnet of the PMSM results in an uneven air-gap flux density, results in unbalanced magnetic pull (UMP), which makes the rotor eccentric, and the eccentricity of the rotor further changes the air-gap flux density of the motor, resulting in UMP changing again. This further illustrates that $F_{M x}(t)$ and $F_{M y}(t)$ are functions related to $x(t), y(t)$ and $F_{\delta}$. So, equation (1) is the relation between the demagnetization parameters $\left(F_{\delta}\right)$ and the eccentricity parameters $(x(t), y(t))$.

Through the combination of equation (1) with equations (12)-(15), the dynamic equation of the magnetic field-rotor coupling system under demagnetization can be obtained: 


$$
M_{r}\{\ddot{x} \ddot{y}\}+C_{r}\left\{\begin{array}{c}
\dot{x} \\
\dot{y}
\end{array}\right\}+K_{r}\left\{\begin{array}{l}
x \\
y
\end{array}\right\}=\left\{\begin{array}{l}
F_{M x} \\
F_{M y}
\end{array}\right\},
$$

where $M_{r}, C_{r}$, and $K_{r}$ are the quality, damping, and stiffness matrix of the rotor system under the demagnetization. $M_{r}=\left[\begin{array}{cc}m & 0 \\ 0 & m\end{array}\right], C_{r}=\left[\begin{array}{cc}c_{x x} & 0 \\ 0 & c_{y y}\end{array}\right]$, and $K_{r}=\left[\begin{array}{cc}k_{x x} & 0 \\ 0 & k_{y y}\end{array}\right]$.

\section{Simulation Calculation and Dynamic Analysis}

In this section, first, we confirm the accuracy of the mathematical model for unbalanced magnetic pull (UMP) under rotor uniform demagnetization and eccentricity by using the mutual verification of Maxwell simulation and MATLAB calculation. Secondly, according to the established dynamic model of PMSMs rotor-magnetic field, the characteristics of the PMSMs rotor system under demagnetization are analyzed. Considering the actual model parameters of PMSM, the parameters are as follows (Table 1).

3.1. Simulation and Verification of UMP Model. For the uniform demagnetization of PMSM, this section focuses on the unbalanced magnetic pull (UMP) model (12) (13) under the condition of uniform demagnetization and eccentricity. The uniform demagnetization and eccentricity of the PMSM are set by Maxwell software, and the results of simulation analysis are verified with MATLAB calculation. The verification mainly considers two aspects:

(i) The accuracy of model (12) and (13)

(ii) Considering the causes of rotor eccentricity (mainly considering whether the rotor eccentricity is completely caused by demagnetization), analyzing the changes of UMP under different eccentric states under the demagnetization

Figure 3 shows the demagnetization model of PMSM by Maxwell. Demagnetization is set in the model with the negative half axis of rotor rectangular coordinate system as the center line. And Figures 4 to 7 can be obtained through corresponding parameters setting and simulation.

In Figure 4, set demagnetization angle $2 * \beta=(\pi / 2)$, the initial eccentric coordinate of the rotor is $(0.2,0.1)$, and set the decrease of air-gap MMF at the demagnetization angle (air-gap MMF drop percentage) $\zeta$ as a variable. The change trend of the resultant force in directions $x, y$ of the UMP under the change of air-gap MMF can be seen that, where the red curve is calculated by MATLAB, and the blue curve is Maxwell data. By comparing the running trend of the two curves, it can be concluded that the two curves are basically consistent.

In Figure 5, set the decrease of air-gap MMF at the demagnetization angle (air-gap MMF drop percentage) $\zeta=50 \%$, the initial eccentric coordinate of the rotor is $(0.2$, 0.1 ), and let demagnetization angle $\beta$ as a variable. We can get from Figure 5 the diagram of resultant force in directions $x, y$ of UMP trend under the change of demagnetization
TABLE 1: The main parameters of PMSM.

\begin{tabular}{lcc}
\hline Variable & Unit & Value \\
\hline Average air-gap length, $\delta_{0}$ & $\mathrm{~mm}$ & 1 \\
Outer radius of the rotor, $r$ & $\mathrm{~mm}$ & 244 \\
Shaft diameter of the rotor, $d$ & $\mathrm{~mm}$ & 52 \\
Effective core length, $l_{0}$ & $\mathrm{~mm}$ & 245 \\
Shaft length, $l$ & $\mathrm{~mm}$ & 410 \\
Number of pole pairs, $p$ & Pair & 2 \\
Phase number of motor, $M_{p}$ & Phase & 3 \\
Coil turn, $N$ & Turn & 10 \\
Rotor quality, $m$ & $\mathrm{~kg}$ & 76 \\
Stator current, $I$ & $\mathrm{~A}$ & 130 \\
Shaft material & & $40 \mathrm{Cr}$ \\
\hline
\end{tabular}

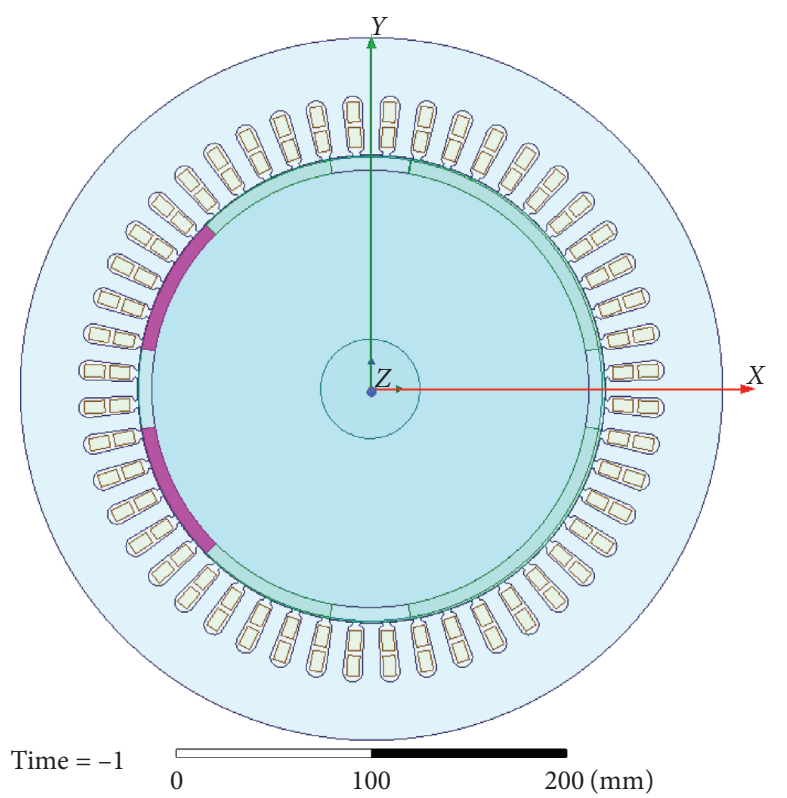

FIGURE 3: The diagram of UMP trend under the change of air-gap MMF.

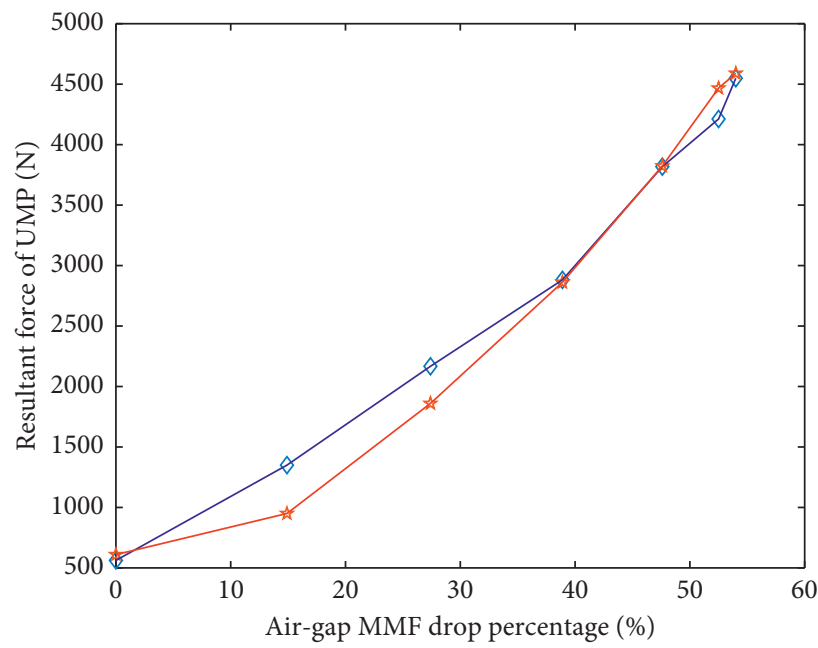

FIGURE 4: The diagram of UMP trend under the change of air-gap MMF. 
angle, where the red curve is calculated by MATLAB according to the mathematical model, and the blue curve is Maxwell data. By comparing the running trend of the two curves, it can be concluded that the two curves are basically consistent.

In Figure 6, set the decrease of air-gap MMF at the demagnetization angle (air-gap MMF drop percentage) $\zeta=50 \%$, the demagnetization angle $2 * \beta=(\pi / 2)$, and the initial eccentric coordinate of the rotor is $(0.2,0.1)$. We can get from Figure 6 the diagram of curve of UMP in directions $x, y$, where the red curve is the UMP in direction $x$, and the blue curve is the UMP in direction $y$. In the figure, the scatter curve is Maxwell simulation data, and continuous curve is MATLAB calculation data. By comparing the data calculated by MATLAB based on mathematical model and Maxwell simulation data, it can be found that the two data are basically consistent.

In Table 2, set the decrease of air-gap MMF at the demagnetization angle (air-gap MMF drop percentage) $\zeta=50 \%$, the demagnetization angle $2 * \beta=(\pi / 2)$. We can get from Table 2 the data table of UMP variation under eccentric direction change.

In Figure 7, set the decrease of air-gap MMF at the demagnetization angle (air-gap MMF drop percentage) $\zeta=50 \%$, the demagnetization angle $2 * \beta=(\pi / 2)$. We can get from Figure 7 the diagram of resultant force in directions $x, y$ of UMP trend under the eccentricity change in direction $x$.

By analyzing Table 2 and Figure 7, it can be concluded that when demagnetization is constant, the UMP on the rotor of PMSM will also change with the change of eccentricity direction and eccentricity.

Therefore, by comparing Figures $4-7$ and the data in Table 2, it can be concluded that the model (12) (13) of UMP under uniform demagnetization and eccentricity can perfectly describe the change of unbalanced magnetic pull (UMP) under different demagnetization states.

\subsection{Vibration Characteristics Analysis of Rotor-Magnetic} Field System. This section mainly analyzes the influence of UMP on the dynamic characteristics of PMSM caused by axial uniform demagnetization of permanent magnet. Considering the rotor eccentricity caused by uniform demagnetization of PMSM and the unbalanced magnetic pull on the rotor, the changes of critical speed and axis orbit of the rotor system are analyzed.

Considering the uniform demagnetization of rotor, the influence of demagnetization angle and decrease of air-gap MMF at the demagnetization angle on rotor dynamic characteristics is studied. In order to further study the dynamic characteristics of rotor under uniform demagnetization, the change of demagnetization angle and decrease of air-gap MMF on rotor dynamic characteristics is studied in this section. Assuming that the initial state is the ideal working state of the motor, the rotor eccentricity caused by the motor assembly is ignored; that is, the rotor coordinate system $O^{\prime}(x, y)$ is consistent with the stator coordinate system $O(x, y)$ in the initial state of PMSM.

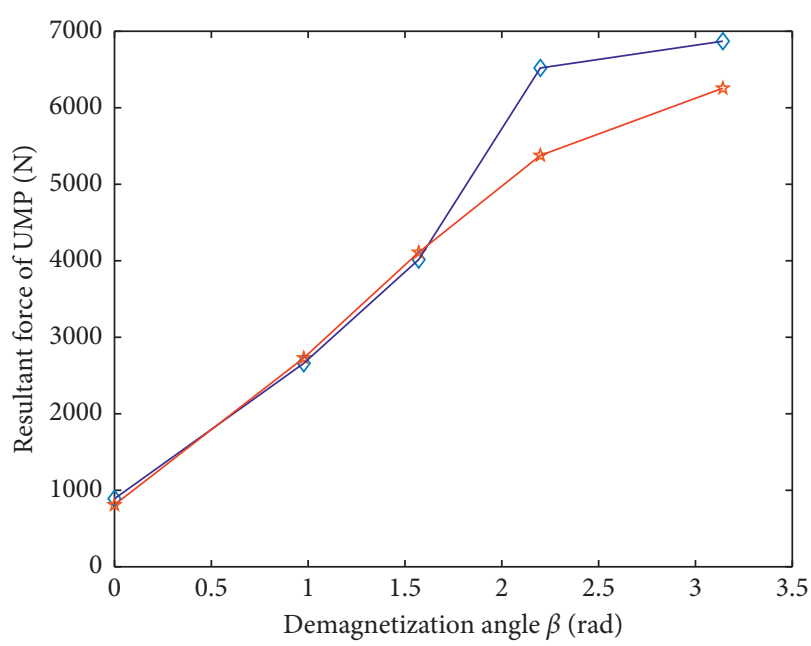

FIgURE 5: The diagram of UMP trend under the change of demagnetization angle.

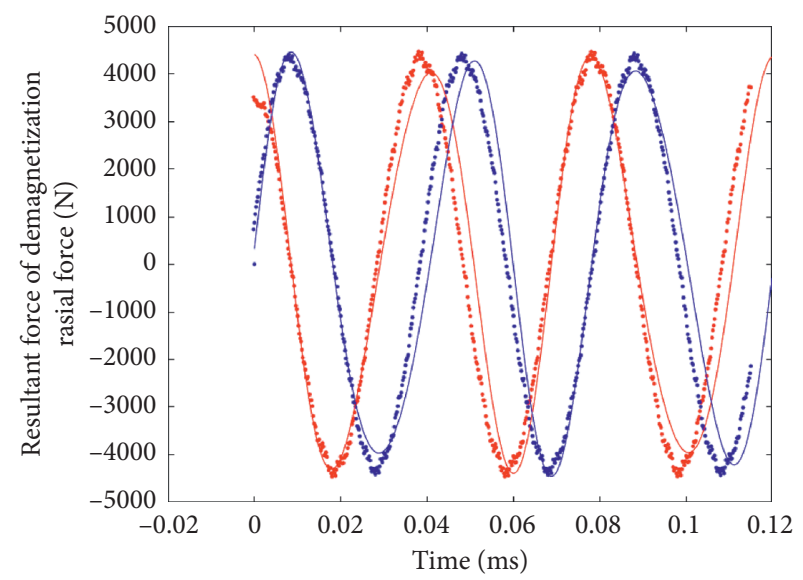

FIgURE 6: The diagram of curve of UMP in directions $x, y$.

TABLE 2: Data table of UMP variation under eccentric direction change.

\begin{tabular}{lcc}
\hline Initial eccentric coordinate & Maxwell & MATLAB \\
\hline $\mathrm{mm}(x, y)$ & Data $(\mathrm{N})$ & Data $(\mathrm{N})$ \\
$(-0.2,0.1)$ & 3355 & 3202 \\
$(0.2,-0.1)$ & 3980 & 3900 \\
$(-0.2,-0.1)$ & 3389 & 3223 \\
$(0.2,0.1)$ & 4050 & 3950 \\
$(0.225,0)$ & 4136 & 4120 \\
$(-0.225,0)$ & 3280 & 3050 \\
$(0,0.225)$ & 3680 & 3628 \\
$(0,-0.225)$ & 3710 & 3625 \\
\hline
\end{tabular}

In this section, the dynamic system is solved by the ode45 solver of MATLAB. Due to the UMP, the system (16) is time-varying differential equations. Set $1 / 300$ of the rotor rotation period as a single step; in a single step, the time variables in the time-varying system (16) are 


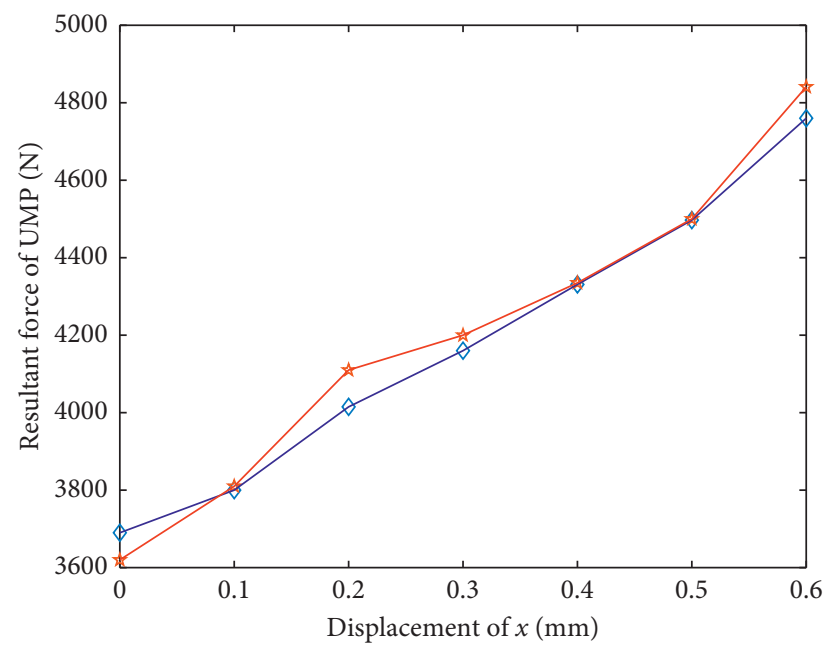

FIgURE 7: The diagram of UMP trend under the eccentricity change in direction $x$.

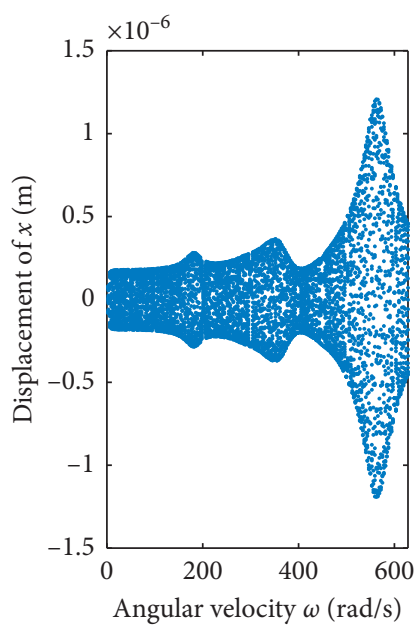

(a)

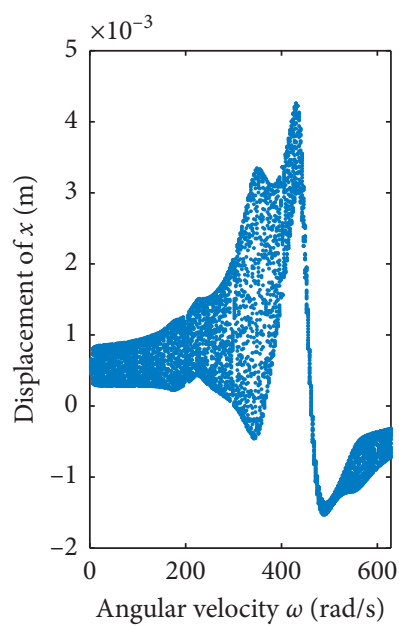

(b)

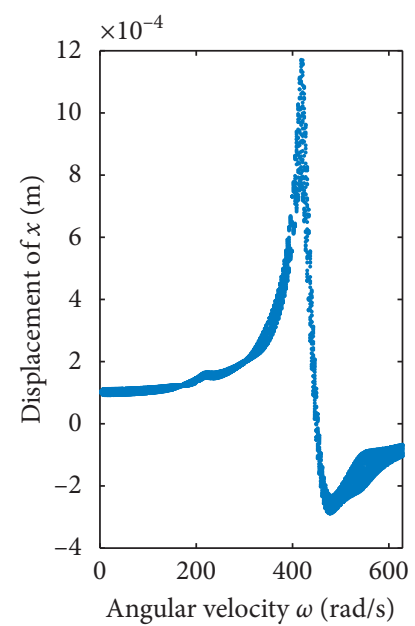

(c)

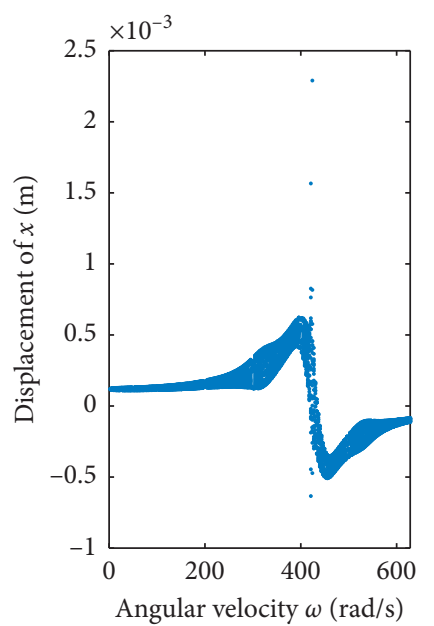

(d)

Figure 8: Bifurcation diagram of rotor system with demagnetization angle variation. (a) $\beta=0$, (b) $\beta=\pi / 6$, (c) $\beta=\pi / 3$, (d) $\beta=\pi / 2$.

linearized by Lipschitz condition, and the ordinary differential equations corresponding to the system (16) under each step are solved in turn, and finally the timevarying differential equation system is solved. Calculate 100 rotation cycles, discard the unsteady state data, taking the solution of steady-state for the next 30 cycles, and discuss the dynamic characteristics of the rotor in the speed $\omega=3.14-628 \mathrm{rad} / \mathrm{s}$.

Figure 8 shows bifurcation diagram of PMSM's rotor system with demagnetization angle variation, setting the decrease of air-gap MMF at the demagnetization angle (airgap MMF drop percentage) is $\zeta=30 \%$, and the demagnetization angle $\beta$ takes $\left[\begin{array}{llll}0 & \pi / 6 & \pi / 3 & \pi / 2\end{array}\right]$.

It can be seen from Figure 8 that the PMSM is normal. When demagnetization angle is at $\beta=0$, at this time, the resultant force of UMP caused by demagnetization is zero, so the amplitude of the PMSM's rotor is small. Compared with
Figure 8, When the decrease of air-gap MMF is fixed, and demagnetization angle $\beta \in(0, \pi / 2]$, with the increase of $\beta$, the nonlinear dynamic characteristics of the rotor system are enhanced, the resonance peak value of the system increases, and the critical speed decreases.

Figure 9 shows bifurcation diagram of rotor system with air-gap MMF drop percentage variation at demagnetization angle, setting the demagnetization angle $2 * \beta=2 * \pi / 15$, and the decrease of air-gap MMF at the demagnetization angle (air-gap MMF drop percentage) $\zeta$ takes $\left[\begin{array}{llll}0 & 20 \% & 40 \% & 60 \%\end{array}\right]$.

It can be seen from Figure 9 that the PMSM is normal. When air-gap MMF drop percentage $\zeta=0$, at this time, the resultant force of UMP caused by demagnetization is zero, so the amplitude of the PMSM's rotor is small. Compared with Figure 9, when the demagnetization angle is fixed, with the increase of the decrease of air-gap MMF at the 


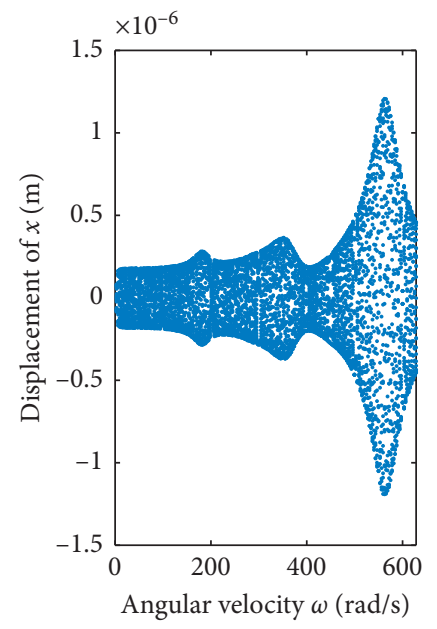

(a)

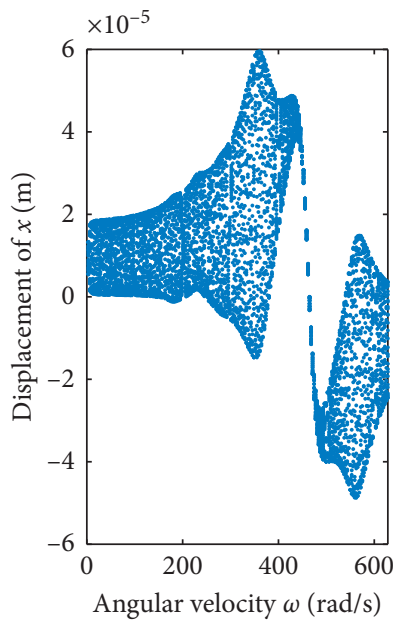

(b)

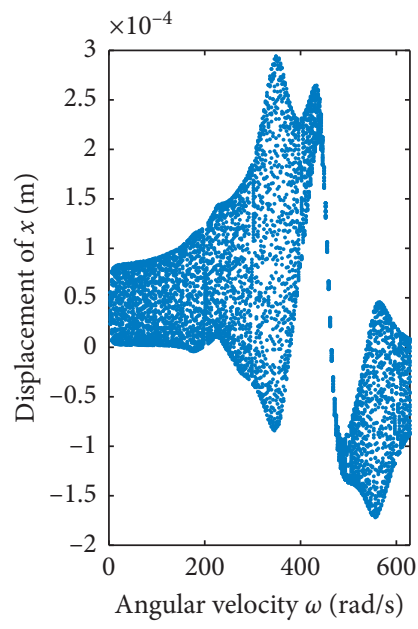

(c)

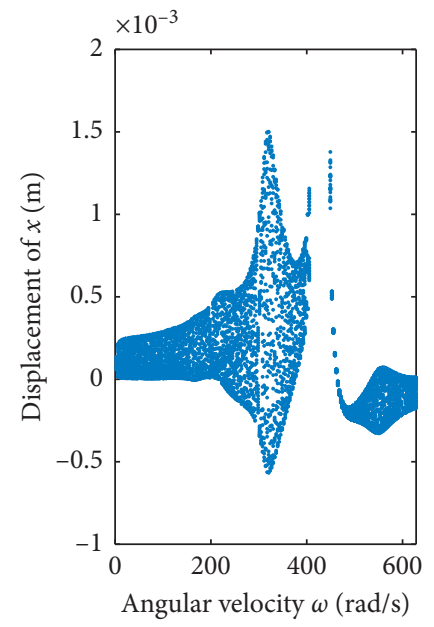

(d)

Figure 9: Bifurcation diagram of rotor system with air-gap MMF drop percentage variation at demagnetization angle. (a) $\zeta=0$, (b) $\zeta=20 \%$, (c) $\zeta=40 \%$, (d) $\zeta=60 \%$.
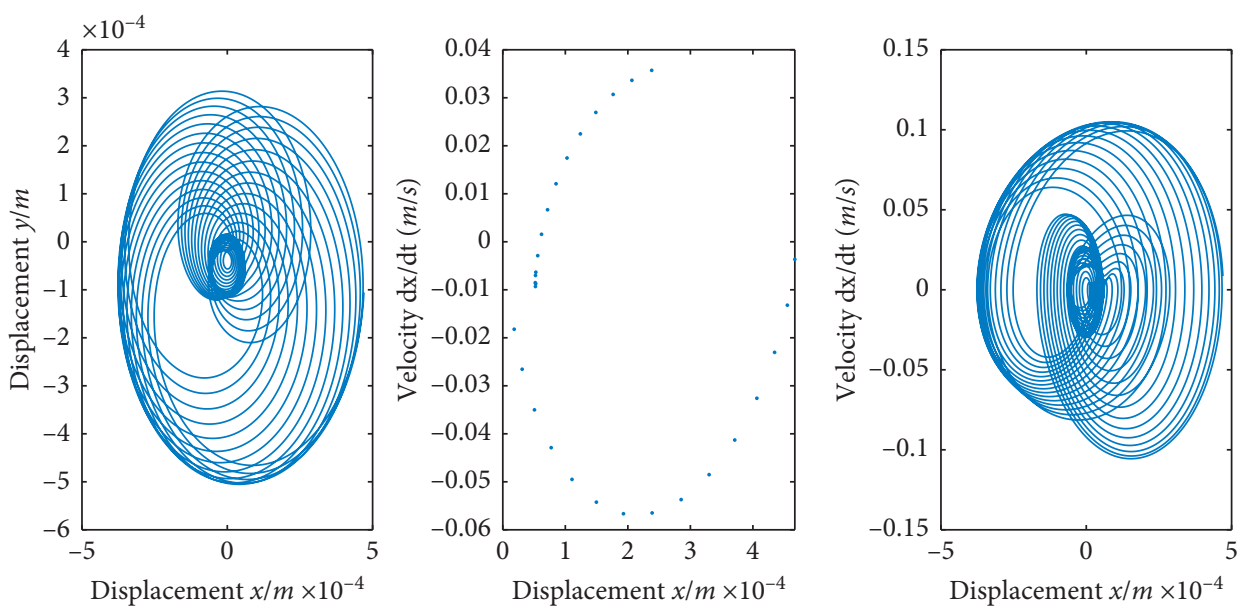

(a)

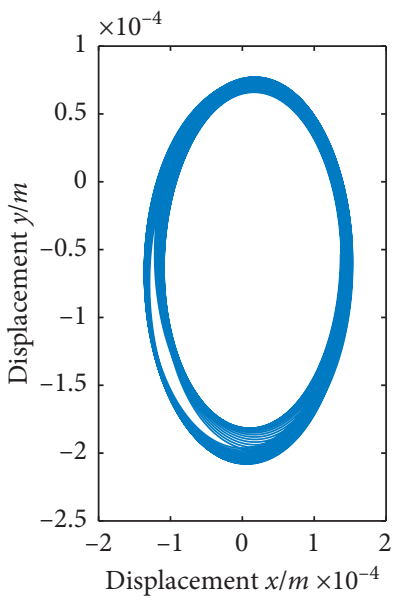

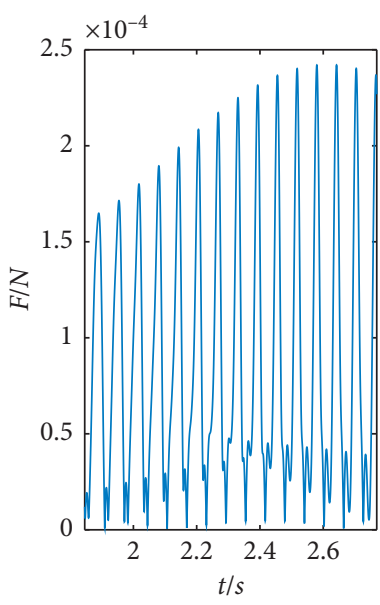
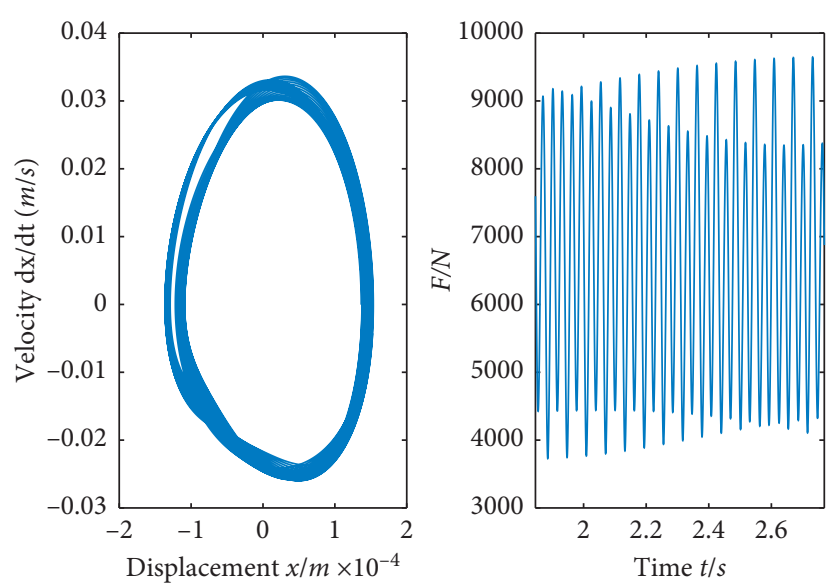

(b)

Figure 10: The rotor operating state diagram under different demagnetization states at $\omega=204.2033 \mathrm{rad} / \mathrm{s}$. (a) Demagnetization states $\zeta=60 \%$ and $\beta=(\pi / 15)$. (b) Demagnetization states $\zeta=30 \%$ and $\beta=(\pi / 3)$. 

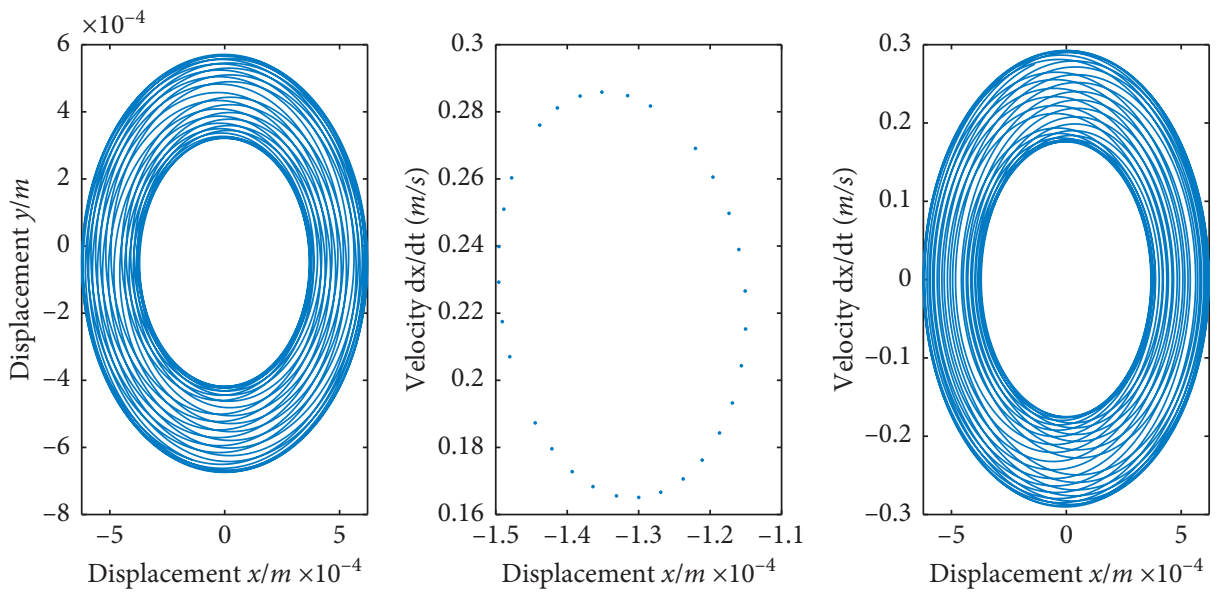

(a)
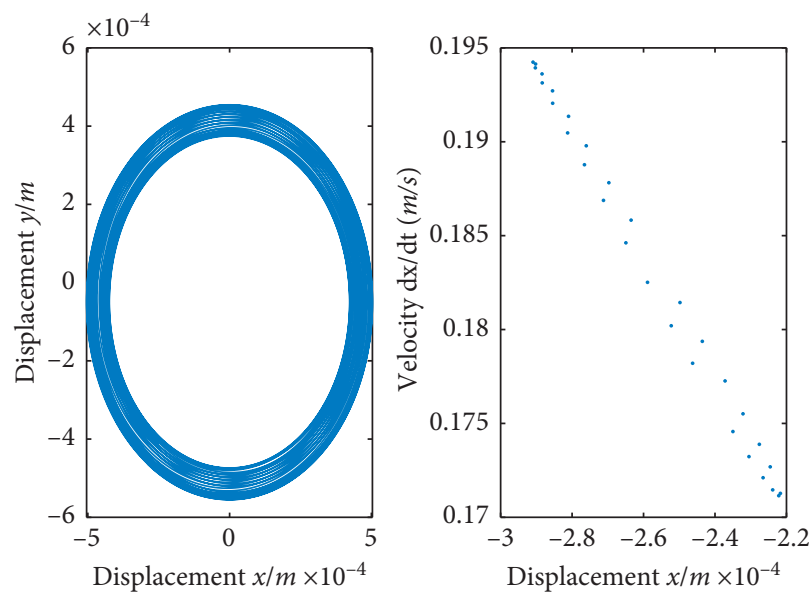
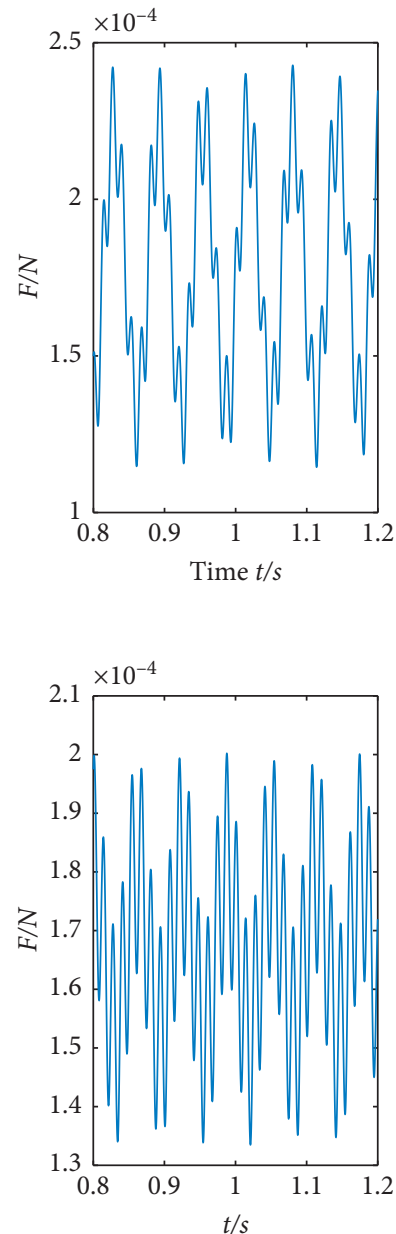

(b)

FIgURE 11: The rotor operating state diagram under different demagnetization states at $\omega=471.2385 \mathrm{rad} / \mathrm{s}$. (a) Demagnetization states $\zeta=60 \%$ and $\beta=(\pi / 15)$. (b) Demagnetization states $\zeta=30 \%$ and $\beta=(\pi / 3)$.

demagnetization angle (air-gap MMF drop percentage) $\zeta$, the nonlinear dynamic characteristics of the rotor system are enhanced, the resonance peak value of the system increases, and the critical speed decreases.

Figure 10 shows the operating state diagram of the rotor under different demagnetization states at $\omega=204.2033 \mathrm{rad} / \mathrm{s}$. Figure 10(a) shows the axis orbit, Poincare, phase plane, and UMP when demagnetization states $\zeta=60 \%$ and $\beta=(\pi / 15)$ are set. Figure 10(b) shows the axis orbit, Poincare, phase plane, and UMP when demagnetization states $\zeta=30 \%$ and $\beta=(\pi / 3)$ are set. The comparison and analysis of the above figures reveal that in the two kinds of demagnetization states the operating state of the rotor is quasiperiodic; however, the axis orbit, Poincare, phase plane, and UMP are different under two demagnetization states.

Figure 11 shows the operating state diagram of the rotor under different demagnetization states at $\omega=471.2385 \mathrm{rad} / \mathrm{s}$. Figure 11(a) shows the axis orbit, Poincare, phase plane, and UMP when demagnetization states $\zeta=60 \%$ and $\beta=(\pi / 15)$ are set. Figure 11(b) shows the axis orbit, Poincare, phase plane, and UMP when demagnetization states $\zeta=30 \%$ and $\beta=(\pi / 3)$ are set. The comparison and analysis of the above figures reveal that in the two kinds of demagnetization states the operating state of the rotor is quasiperiodic; however, the axis orbit, Poincare, phase plane, and UMP are different under two demagnetization states.

By comparing Figures $8-11$, it can be found that the influence of different demagnetization states and speeds on the dynamic characteristics of PMSM's rotor is different.

\section{Conclusion}

In this paper, the rotor-magnetic field coupling dynamics Jeffcott model of permanent magnet synchronous motor (PMSM) under axial uniform demagnetization is considered. On this basis, the demagnetization status of permanent magnet in PMSM is described from two aspects of demagnetization angle and decrease of air-gap MMF at the demagnetization angle (air-gap MMF drop percentage), and the influence of demagnetization on the operation of rotor system is analyzed. This study provides a theoretical basis for the accurate fault diagnosis and vibration control of PMSMs in the future. 
(i) In view of the UMP produced by the uniform demagnetization of PMSM causing the rotor eccentricity to change the air-gap flux density of the PMSM again, in this paper, the PMSM is modeled and simulated by Maxwell software. By setting the demagnetization state and eccentricity state in the model, the UMP data under different uniform demagnetization and eccentricity states are obtained by simulation. The UMP data generated by Maxwell is compared with the MATLAB data calculated based on the mathematical model under the same uniform demagnetization and eccentricity conditions in this paper, so as to determine the validity of the UMP model under uniform demagnetization and eccentricity.

Meanwhile, this paper simulates by setting different eccentric states (eccentric magnitude, eccentric angle) of the rotor under the same demagnetization state. By comparing the UMP data, it can be seen that the UMP generated by the same demagnetization state and different rotor eccentricity state has great difference. Therefore, combined with the model (16) established in this paper, it can provide a theoretical basis for the judgment of demagnetization and other mixed faults of PMSM.

(ii) Based on the rotor-magnetic field coupling dynamics model of PMSM under uniform demagnetization, analyzing the influence of demagnetization of the rotor permanent magnet on the operation of the rotor system, the following conclusions can be drawn:

When the decrease of air-gap MMF is fixed, and demagnetization angle $\beta \in(0, \pi / 2]$, with the increase of $\beta$, the nonlinear dynamic characteristics of the rotor system are enhanced, the resonance peak value increases, and the critical speed decreases.

When the demagnetization angle is fixed, with the increase of decrease of air-gap MMF at the demagnetization angle (air-gap MMF drop percentage) $\zeta$, the nonlinear dynamic characteristics of the rotor system are enhanced, the resonance peak value increases, and the critical speed decreases.

In different demagnetization states (demagnetization angle, decrease of air-gap MMF), the axis orbit, Poincare, phase plane, and UMP have unique characteristics. So, the demagnetization state can be reflected from the axis orbit, Poincare, phase plane, and UMP, which provides a theoretical basis for the accurate fault diagnosis and vibration control of PMSMs in the future.

\section{Data Availability}

The data used to support the findings of this study are included within the article.

\section{Conflicts of Interest}

The authors declare that they have no conflicts of interest.

\section{Acknowledgments}

This work was supported by the National Natural Science Foundation of China (11972156).

\section{References}

[1] W. P. Lu, H. Zhao, and Y. L. Siluo, "Analysis of demagnetization field of line-start permanent magnet synchronous motor under abnormal operation conditions," Electric Machines and Control, vol. 17, no. 7, pp. 7-14, 2013.

[2] T. Xu, X. Wang, L. I. Ying et al., "Demagnetization study for line-start permanent magnet synchronous motor during starting process," Proceedings of the CSEE, vol. 35, no. 4, pp. 961-970, 2015.

[3] G. Choi and T. M. Jahns, "Investigation of key factors influencing the response of permanent magnet synchronous machines to three-phase symmetrical short-circuit faults," IEEE Transactions on Energy Conversion, vol. 31, no. 4, pp. 1488-1497, 2016.

[4] M. Zhang, X. Xiao, and L. I. Yong-Dong, "Speed and flux linkage observer for permanent magnet synchronous motor based on EKF," Proceedings of the CSEE, vol. 27, no. 36, pp. 36-40, 2007.

[5] W. Hai-Feng, W. Han-Pei, Y. Zhang et al., "New stator flflux observer of permanent magnet synchronous motor considering stator resistance perturbation under virtual $\gamma-\delta$ rotating coordinate system," Control and Decision, vol. 32, no. 12, pp. 2301-2304, 2017.

[6] G. Feng, C. Lai, K. Mukherjee, and N. C. Kar, "Online PMSM magnet flux-linkage estimation for rotor magnet condition monitoring using measured speed harmonics," IEEE Transactions on Industry Applications, vol. 53, no. 3, pp. 2786-2794, 2017.

[7] F. Hu, D. Luo, C. Luo, Z. Long, and G. Wu, "Cascaded robust fault-tolerant predictive control for PMSM drives," Energies, vol. 11, no. 11, p. 3087, 2018.

[8] C. Zhang, G. Wu, F. Rong et al., "Robust fault-tolerant predictive current control for permanent magnet synchronous motors considering demagnetization fault," IEEE Transactions on Industrial Electronics, vol. 65, no. 7, pp. 5324-5334, 2018.

[9] C. Zhang, G. Wu, J. He et al., "fault-tolerant predictive control for demagnetization faults in permanent magnet synchronous machine," Transactions of china Electro Technical Society, vol. 32, no. 15, pp. 100-110, 2017.

[10] H. He, N. Zhou, J. Guo, Z. Zhang, B. Lu, and C. Sun, “Tolerance analysis of electrified vehicles on the motor demagnetization fault: from an energy perspective," Applied Energy, vol. 227, pp. 239-248, 2018.

[11] J. De Bisschop, A. Abdallh, P. Sergeant, and L. Dupre, "Identification of demagnetization faults in axial flux permanent magnet synchronous machines using an inverse problem coupled with an analytical model," IEEE Transactions on Magnetics, vol. 50, no. 11, pp. 1-4, 2014.

[12] I. Coenen, M. Van Der Giet, and K. Hameyer, "Manufacturing tolerances: estimation and prediction of cogging torque influenced by magnetization faults," IEEE Transactions on Magnetics, vol. 48, no. 5, pp. 1932-1936, 2012.

[13] J. D. McFarland and T. M. Jahns, "Investigation of the rotor demagnetization characteristics of interior PM synchronous machines during fault conditions," IEEE Transactions on Industry Applications, vol. 50, no. 4, pp. 2768-2775, 2014. 
[14] S. Jikai, L. Zhang, F. Haichao et al., "Characteristic analysis and optimization of line-start surface-mounted and interior permanent magnet synchronous motor," Transactions of china Electro Technical Society, vol. 32, no. 9, pp. 63-69, 2017.

[15] T. Ishikawa, Y. Seki, and N. Kurita, "Analysis for fault detection of vector-controlled permanent magnet synchronous motor with permanent magnet defect," IEEE Transactions on Magnetics, vol. 49, no. 5, pp. 2331-2334, 2013.

[16] L. Cui, Yu Zhang, X. Gong et al., "Vibration mechanism based quantitative diagnosis and quantization analysis of rolling bearing fault," Journal of Beijing University of Technology, vol. 41, no. 11, pp. 1681-1687, 2015.

[17] J. Qiu, Nonlinear Vibration of Electromechanical Coupled Dynamic System, Science Press, Beijing, China, 1996.

[18] Z. Qiu, Z. Deng, X. Wang, and L. Meng, "Study on the modeling and control of a permanent-magnet-type bearingless motor considering rotor eccentricity and Lorenz force," Proceedings of the CSEE, vol. 27, no. 9, pp. 64-70, 2007. 